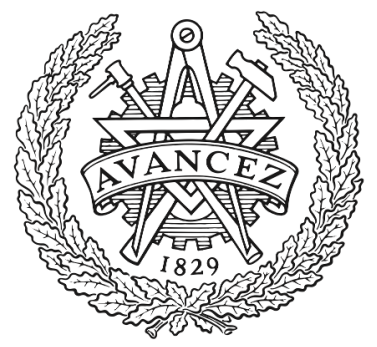

CHALMERS

UNIVERSITY OF TECHNOLOGY

\title{
The closest isotropic, cubic and transversely isotropic stiffness and compliance tensor to an arbitrary anisotropic material
}

Downloaded from: https://research.chalmers.se, 2023-04-26 05:40 UTC

Citation for the original published paper (version of record):

Shao, X., Folkow, P., Eskandari-Ghadi, M. (2021). The closest isotropic, cubic and transversely isotropic stiffness and compliance tensor to an arbitrary anisotropic material. Journal of Mechanics of Materials and Structures, 16(4): 451-470. http://dx.doi.org/10.2140/jomms.2021.16.451

N.B. When citing this work, cite the original published paper. 


\section{Journal of}

\section{Mechanics of}

\section{Materials and Structures}

THE CLOSEST ISOTROPIC, CUBIC AND TRANSVERSELY ISOTROPIC STIFFNESS AND COMPLIANCE TENSOR TO AN ARBITRARY ANISOTROPIC MATERTAL

Xinyuan Shao, Peter D. Folkow and Morteza Eskandari-Ghadi

\section{1}

Volume 16, No. 4

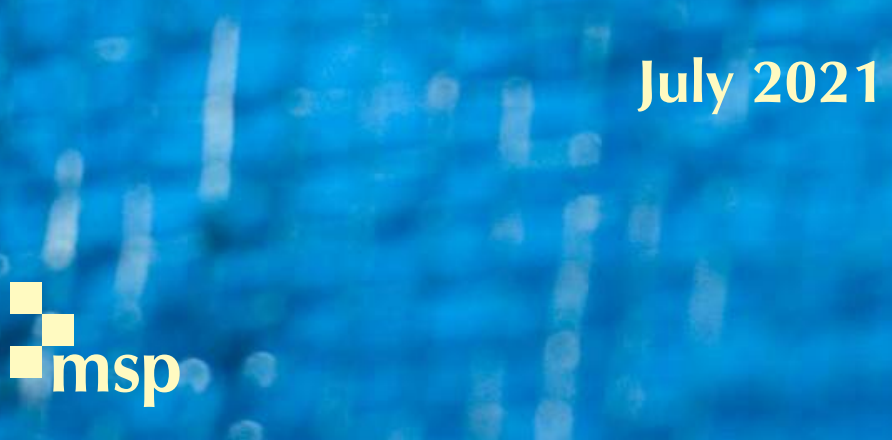




\title{
THE CLOSEST ISOTROPIC, CUBIC AND TRANSVERSELY ISOTROPIC STIFFNESS AND COMPLIANCE TENSOR TO AN ARBITRARY ANISOTROPIC MATERIAL
}

\author{
Xinyuan Shao, Peter D. Folkow and Morteza Eskandari-Ghadi
}

\begin{abstract}
The aim of this paper is to provide, in the framework of Green elasticity, the closest or nearest fourthorder isotropic, cubic and transversely isotropic elasticity tensors with higher symmetries for a general anisotropic elasticity tensor or any other tensors with lower symmetry. Using a gauge parameter, the procedure is done on a dimensionless form based on different generalized Euclidean distances, namely conventional, log-, and power-Euclidean distance functions. In the case of power-Euclidean distance functions, results are presented for powers of 0.5, 1 and 2. Except for the conventional distance function, the different generalized distance functions adopted in this paper preserve the property of invariance by inversion, meaning that the results for the closest stiffness tensor are also valid for the compliance tensor. Explicit formulations are given for determining the closest isotropic and cubic tensors, where the multiplication tables of the bases are diagonal. More involved coupled equations are given for the coefficients of the closest transversely isotropic elasticity tensors, which can be solved numerically. Two different material cases are studied in the numerical examples, which illustrate the material coefficients and error measures based on the present methods, including the influence from the gauge parameter.
\end{abstract}

\section{Introduction}

Analytical solutions of engineering problems provide means for deep understanding for the phenomena involved. Because of this, both engineers and applied mathematicians are interested in analytical solutions for elastostatics and elastodynamics boundary value problems. However, determining analytical solutions are difficult in many cases. One of the reasons is related to complicated constitutive law governing the behaviour of the natural and artificial engineering materials. In mechanics, anisotropy is natural rather than being exceptions. Dealing with general anisotropic material in elastostatics and elastodynamics results in some complicated systems of partial differential equations that cannot be solved analytically, even for simple problems. Therefore, finding the closest isotropic material, or anisotropic material with certain symmetry properties (cubic, transversely isotropic), to the original anisotropic material with lower symmetry is promising. By replacing the general anisotropic constitutive law with constitutive law containing upper symmetry, one may find analytical solutions for various boundary value problems.

One of the applications of determining the closest tensor with upper symmetry to the general anisotropic tensor is for wave propagation phenomena, where the speed of different waves traveling in a particular direction in an anisotropic material is of interest. Norris [2006b], with the use of conventional Euclidean distance function, gave answers to Fedorov question: "What elastically isotropic material is the best

Keywords: conventional distance, log-distance, power-Euclidean distance, closest elasticity tensor, linear vector space. 
acoustic fit to a given set of anisotropic moduli?" He proved that the solution based on minimizing a Euclidean distance function is identical to the method of minimizing the mean-squared difference.

Linear vector space is a powerful tool for preparing a solution platform, where one may define distance functions between general vectors. This allows to find the closest vector to a given general vector. As the closest vector from a subspace to a general vector in a vector space is dependent on the distance function used, different distance functions may result in different closest vectors. On the other hand, the constitutive law in mechanics may be given in terms of either stiffness or compliance tensors. Thus, the inverse of the closest stiffness tensor should also be the closest compliance tensor. One may hereby define an inner product with the usual properties accompanied with the property of invariance under inversion; see Morin et al. [2020].

Gazis et al. [1963] were, to the best knowledge of the authors, the first who used an inner product space to present the closest isotropic tensor to an arbitrary fourth-order tensor in the subject of continuum mechanics and elasticity. They used the conventional Euclidean (also named Frobenius) distance function for their investigation. Norris [2006a] presented an algorithm for determining the closest isotropic tensors to a given material of arbitrary symmetry, based on three different distance functions: the Frobenius, the Riemannian, and the log-Euclidean distance functions. Moakher and Norris [2006] applied three different distance functions (Frobenius, Riemannian and log-Euclidean distance) and presented algorithms for determining the best fourth-order tensor with arbitrary symmetry selected from a set of ten different symmetries to an elasticity tensor of lower symmetry, where they used Walpole algebraic tensor decomposition to represent bases for elastic material with different symmetries.

The conventional Euclidean distance function is not invariant under inversion of the elasticity tensor. However, the Riemannian distance function [Moakher 2006] and the log-Euclidean distance function [Arsigny et al. 2005] are invariant under inversion. On the other hand, the stiffness/compliance of an elastic material is not dimensionless, and the distance function may be influenced by the order (values) of the material properties [Morin et al. 2020]. Morin et al. [2020] modified different distance functions as to make them dimensionless. To this end, they normalized the elasticity tensor by a gauge parameter with the dimension of stiffness, where they showed that the closest isotropic tensor based on log-distance function is independent from the parameter while the other distance functions, say arc-tan and power distance functions, depend on that parameter.

In this paper, we present the closest isotropic, cubic and transversely isotropic fourth-order material tensors to arbitrary general anisotropic tensors with the use of conventional, log-, and power-Euclidean distance functions in the framework of Green-elasticity. In order to make dimensionless argument for different distance functions, a gauge parameter with the dimension of stiffness is introduced as proposed in [Morin et al. 2020]. The present work is a continuation to the studies on closest isotropic material in that reference, by including the closest anisotropic materials with cubic and transversely isotropic symmetries. Explicit solution processes are given for these simplified material configurations, and numerical results based on two types of anisotropic materials are presented; one orthotropic material and one more general anisotropic material. 


\section{The general theory}

This paper considers elasticity tensors defining so called Green-elastic linear materials. Consequently, the material modelling based on fourth-order tensors having both major and minor symmetries, may be equivalently expressed using second-order symmetric tensors in Kelvin notation. More specifically, a general fourth-order elastic tensor $\mathbb{C}$ with components $C_{i j k l}$ in the given Cartesian coordinate system $x_{1} x_{2} x_{3}$, may be expressed using the equivalent second-order tensor based on Kelvin notation $\hat{\mathbf{C}}$ with components $\hat{c}_{I J}$. The component relations are $C_{i j k l} \equiv \hat{c}_{I J}$, if $I$ or $J=1,2,3,4,5$ and 6 correspond to $i j$ or $k l=11,22,33,23,13$ and 12 , respectively. In addition, we use a normalization factor of $\sqrt{2}$ for off-diagonal $3 \times 3$ sub-matrices. Thus, the Kelvin notation of the elastic moduli is shown by [Morin et al. 2020; Moakher and Norris 2006]

$$
\hat{\mathbf{C}}=\left(\begin{array}{cccccc}
c_{11} & c_{12} & c_{13} & \sqrt{2} c_{14} & \sqrt{2} c_{15} & \sqrt{2} c_{16} \\
c_{12} & c_{22} & c_{23} & \sqrt{2} c_{24} & \sqrt{2} c_{25} & \sqrt{2} c_{26} \\
c_{13} & c_{23} & c_{33} & \sqrt{2} c_{34} & \sqrt{2} c_{35} & \sqrt{2} c_{36} \\
\sqrt{2} c_{14} & \sqrt{2} c_{24} & \sqrt{2} c_{34} & 2 c_{44} & 2 c_{45} & 2 c_{46} \\
\sqrt{2} c_{15} & \sqrt{2} c_{25} & \sqrt{2} c_{35} & 2 c_{45} & 2 c_{55} & 2 c_{56} \\
\sqrt{2} c_{16} & \sqrt{2} c_{26} & \sqrt{2} c_{36} & 2 c_{46} & 2 c_{56} & 2 c_{66}
\end{array}\right) .
$$

In order to find the closest tensor with upper symmetry in the sense of group theory for a given tensor of lower symmetry or a general anisotropic Green-elastic material, we need to equip the pertinent linear vector space with an appropriate inner product. In this context, a prerequisite for such a linear vector space is that it should be equipped with a norm. With the norm defined for the vector space, one may thus calculate distances from one tensor to another in the original linear vector space. In this paper, we adopt the distance based on conventional inner product as well as some different $f$-Euclidean distances for the linear vector space [Morin et al. 2020; Moakher and Norris 2006; Moakher 2006]. The scalar-valued functions $f$, which define different distances, are strictly monotone functions which can be constructed based on other continuously differentiable functions. Here, we chose log-, $n$-power- ( $n=1$ and 2 ), and square root functions, to define different distances.

The inner product of two fourth-order tensors $\mathbb{C}$ and $\mathbb{D}$ is denoted by $\langle\mathbb{C}, \mathbb{D}\rangle$. Equivalently, the inner product of two second-order tensors $\hat{\mathbf{C}}$ and $\hat{\mathbf{D}}$ is denoted by $\langle\hat{\mathbf{C}}, \hat{\mathbf{D}}\rangle$. Based on the required properties (symmetry, linearity, positive definiteness) the inner product using Kelvin notation is expressed as

$$
\langle\mathbb{C}, \mathbb{D}\rangle=\langle\hat{\mathbf{C}}, \hat{\mathbf{D}}\rangle=\hat{c}_{I J} \hat{d}_{I J},
$$

from which the norm $\|\mathbb{C}\|$ of the tensor $\mathbb{C}$ is obtained from the property of positive definiteness as

$$
\|\mathbb{C}\| \equiv\|\hat{\mathbf{C}}\|=\sqrt{\hat{\mathbf{C}}: \hat{\mathbf{C}}}=\sqrt{\hat{c}_{I J} \hat{c}_{I J}} .
$$

2A. Euclidean distances for elasticity tensors. From the definition of a norm, one may define distance functions between tensors that should satisfy certain properties. For tensors $\mathbf{M}_{\mathbf{1}}, \mathbf{M}_{\mathbf{2}}$ and $\mathbf{M}_{\mathbf{3}}$ these properties are [Deza and Deza 2009]:

- Nonnegativity:

$$
d\left(\mathbf{M}_{1}, \mathbf{M}_{2}\right) \geq 0,
$$


where equality is valid if and only if $\mathbf{M}_{\mathbf{1}}=\mathbf{M}_{\mathbf{2}}$.

- Symmetry:

$$
d\left(\mathbf{M}_{1}, \mathbf{M}_{2}\right)=d\left(\mathbf{M}_{2}, \mathbf{M}_{1}\right)
$$

- Triangle inequality:

$$
d\left(\mathbf{M}_{1}, \mathbf{M}_{2}\right) \leq d\left(\mathbf{M}_{\mathbf{1}}, \mathbf{M}_{3}\right)+d\left(\mathbf{M}_{\mathbf{2}}, \mathbf{M}_{3}\right) .
$$

Moreover, the distance function in elasticity must be invariant under the operation of inversion in order not to affect the constitutive law expressed using stiffness or compliance tensors, which means

$$
d\left(\mathbf{M}_{\mathbf{1}}, \mathbf{M}_{\mathbf{2}}\right)=d\left(\mathbf{M}_{\mathbf{1}}^{-1}, \mathbf{M}_{\mathbf{2}}^{-1}\right) .
$$

2B. Some definitions of distances. As mentioned earlier, we consider three different distance functions in this paper. The conventional Euclidean distance and two function related Euclidean distances, namely log-Euclidean and power-Euclidean distances. These are defined as:

- The conventional Euclidean distance [Norris 2006a]

$$
d_{C}(\mathbf{A}, \mathbf{B})=\|\mathbf{A}-\mathbf{B}\| .
$$

- The log-Euclidean distance [Arsigny et al. 2005]

$$
d_{L}(\mathbf{A}, \mathbf{B})=\|\ln (\mathbf{A})-\ln (\mathbf{B})\| .
$$

- The power-Euclidean distance [Morin et al. 2020]

$$
d_{P}(\mathbf{A}, \mathbf{B})=\left\|\mathbf{A}^{n}-\mathbf{B}^{n}+\mathbf{B}^{-n}-\mathbf{A}^{-n}\right\| .
$$

Note that (2-8)-(2-10) can be expressed as

$$
d_{f}(\mathbf{A}, \mathbf{B})=\|\boldsymbol{f}(\mathbf{A})-\boldsymbol{f}(\mathbf{B})\|,
$$

where $f$ is a tensor function on a tensor set. Using from now on the Kelvin notation, the functions $f$ are to be established on positive definite $6 \times 6$ matrices [Morin et al. 2020]. To do so, we may define $f(\mathbf{D})$ for a diagonal $6 \times 6$ matrix $\mathbf{D}$ to be a diagonal $6 \times 6$ matrix $\tilde{\mathbf{D}}$, whose diagonal elements $\tilde{d}_{I I}$ are determined by $f\left(d_{I I}\right)$. Thus, the matrix $\tilde{\mathbf{A}}=\boldsymbol{f}(\mathbf{A})$ may be determined by taking the following steps:

- Performing a diagonalization of matrix $\mathbf{A}=\mathbf{R}^{\mathbf{T}} \mathbf{D R}$ and obtain $\mathbf{D}$.

- Applying the function to the diagonal elements of $\mathbf{D}$, which results in $\tilde{\mathbf{D}}$.

- Obtaining the function $f(\mathbf{A})$ by $\tilde{\mathbf{A}}=f(\mathbf{A})=\mathbf{R}^{\mathrm{T}} \tilde{\mathbf{D}} \mathbf{R}$,

where $\mathbf{D}$ is the the diagonal matrix containing the eigenvalues of the original matrix $\mathbf{A}$, and $\mathbf{R}$ is the rotational matrix containing the eigenvectors of $\mathbf{A}$.

Thus, the different distance functions of interest here are based on the elementary functions:

- For the conventional Euclidean distance

$$
f(x)=x .
$$

- For the log-Euclidean distance

$$
f(x)=\ln (x) .
$$


- For the power-Euclidean distance

$$
f(x)=x^{n}-x^{-n}
$$

where $n$ is a real number excluding zero. We should notice that since the elasticity tensor is a positive definite tensor, its eigenvalues are positive and thus the log-Euclidean distance keep the positive definiteness property of the original tensor. In addition, the power-Euclidean distance is a monotonic function based on the defined power function given in (2-14) (see also [Morin et al. 2020]).

\section{Closest tensors with upper symmetry to tensors of lower symmetry}

This section states the fundamentals for deriving the closest isotropic, cubic and transversely isotropic elastic tensors to a given elastic tensor with arbitrary symmetry. To this end, we adopt the basic tensor algebra presented by Walpole [1984] for making bases for these higher symmetry sub-spaces.

3A. Closest isotropic material for arbitrary anisotropic material. The main part of this section has in essence already been presented by Morin et al. [2020], but is given below with some more details for completeness.

A general isotropic fourth-order tensor is given by a linear combination of any two linear independent isotropic tensors, given as bases for linear vector space of isotropic tensors. Following Walpole [1984] (see also [Morin et al. 2020] and [Moakher and Norris 2006]), we select $\mathbb{V}$ and $\mathbb{K}$, which are defined shortly, as a bases here, whose multiplication table is diagonal. Using Kelvin notation, any arbitrary isotropic tensor may be written as a linear combination of $\hat{\mathbf{J}}$ and $\hat{\mathbf{K}}$ :

$$
\hat{\mathbf{A}}=a \hat{\mathbf{J}}+b \hat{\mathbf{K}} \text {. }
$$

In order to define the tensors $\hat{\mathbf{J}}$ and $\hat{\mathbf{K}}$ in a given Cartesian coordinate system $x_{1} x_{2} x_{3}$, we define the unit vector $\mathbf{u}$ in the form of [Walpole 1984]

$$
\mathbf{u}=\left(\begin{array}{llllll}
\frac{1}{\sqrt{3}} & \frac{1}{\sqrt{3}} & \frac{1}{\sqrt{3}} & 0 & 0 & 0
\end{array}\right)^{\mathbf{T}}
$$

which is used to construct the $6 \times 6$ matrix $\hat{\mathbf{J}}$ as

$$
\hat{\mathbf{J}}=\mathbf{u u}^{\mathbf{T}} .
$$

Then the matrix $\hat{\mathbf{K}}$ independent from $\hat{\mathbf{J}}$ is given by

$$
\hat{\mathbf{K}}=\hat{\mathbf{I}}-\hat{\mathbf{J}},
$$

in which the unit $6 \times 6$ tensor $\hat{\mathbf{I}}$ is

$$
\hat{\mathbf{I}}=\operatorname{diag}(1,1,1,1,1,1) .
$$

Specifically, any isotropic elasticity tensor is written in the form of [Walpole 1984]

$$
\hat{\mathbf{C}}_{\text {iso }}=3 \kappa \hat{\mathbf{J}}+2 \mu \hat{\mathbf{K}},
$$

in which $\kappa$ and $\mu$ are the bulk and shear moduli for isotropic material. 
Based on the different distance functions given in the previous section, one may find the distance between two given tensors. Select one of those tensors to be an arbitrary isotropic tensor, then the distance will be a two-variable scalar-valued real function. The nearest isotropic tensor to the given tensor is determined by minimizing this two-variable real distance function. The mathematical details on this part can be found in [Morin et al. 2020].

Since the elements of elasticity tensor are not dimensionless, a gauge with a unit of the elements of elasticity tensor, namely $E_{0}$, can be used to get a nondimensional elastic tensor [Morin et al. 2020]. Then the $f$-distance between an arbitrary elastic tensor, $\hat{\mathbf{C}}$, to an isotropic elastic tensor is calculated as

$$
d_{f}=\left\|f\left(\frac{\hat{\mathbf{C}}}{E_{0}}\right)-f\left(\frac{\hat{\mathbf{C}}_{\text {iso }}}{E_{0}}\right)\right\|,
$$

where $\hat{\mathbf{C}}_{\text {iso }}$ is the closest isotropic elasticity tensor we are looking for. With the definition of the $f$-function, $\boldsymbol{f}\left(\hat{\mathbf{C}}_{\text {iso }}\right)$ is an isotropic elastic tensor which is also a linear combination of the bases

$$
\boldsymbol{f}\left(\hat{\mathbf{C}}_{\mathrm{iso}}\right)=f(3 \kappa) \hat{\mathbf{J}}+f(2 \mu) \hat{\mathbf{K}} .
$$

Similarly

$$
\boldsymbol{f}\left(\frac{\hat{\mathbf{C}}_{\text {iso }}}{E_{0}}\right)=f\left(\frac{3 \kappa}{E_{0}}\right) \hat{\mathbf{J}}+f\left(\frac{2 \mu}{E_{0}}\right) \hat{\mathbf{K}}
$$

Substituting (3-9) into (3-7) results in

$$
d_{f}=\left\|f\left(\frac{\hat{\mathbf{C}}}{E_{0}}\right)-f\left(\frac{3 \kappa}{E_{0}}\right) \hat{\mathbf{J}}-f\left(\frac{2 \mu}{E_{0}}\right) \hat{\mathbf{K}}\right\| .
$$

Looking for the minimum of $d_{f}$ or equally the minimum of $d_{f}^{2}$, we may write

$$
\frac{\partial\left(d_{f}^{2}\right)}{\partial \kappa}=0, \quad \frac{\partial\left(d_{f}^{2}\right)}{\partial \mu}=0,
$$

from which [Morin et al. 2020]

$$
f\left(\frac{3 \kappa}{E_{0}}\right)=f\left(\frac{\widehat{\mathbf{C}}}{E_{0}}\right): \widehat{\mathbf{J}}, \quad f\left(\frac{2 \mu}{E_{0}}\right)=\frac{1}{5} f\left(\frac{\widehat{\mathrm{C}}}{E_{0}}\right): \widehat{\mathrm{K}} .
$$

The function $f$ is a strictly monotone function, thus is one-to-one, and its inverse exists. Applying the inverse function, $f^{-1}$, results in the parameters $\kappa$ and $\mu$ :

$$
\kappa=\frac{E_{0}}{3} f^{-1}\left(f\left(\frac{\hat{\mathbf{C}}}{E_{0}}\right): \hat{\mathbf{J}}\right), \quad \mu=\frac{E_{0}}{2} f^{-1}\left(\frac{1}{5} f\left(\frac{\hat{\mathbf{C}}}{E_{0}}\right): \hat{\mathbf{K}}\right) .
$$

From the definition of the logarithm of a positive definitive symmetric tensor $\hat{\mathbf{A}}$, one may use the relation $\ln (\beta \hat{\mathbf{A}})=\ln (\beta) \hat{\mathbf{I}}+\ln (\hat{\mathbf{A}})$, which is valid for $\forall \beta>0$, to show that the values of $\kappa$ and $\mu$ are independent from the gauge parameter $E_{0}$ for log-distance function, while their values are $E_{0}$-dependent for powerdistance functions. 
3B. Closest cubic material for arbitrary anisotropic material. As the three crystallographic direction of the cubic system are perpendicular [Walpole 1984], a third symmetric tensor linearly independent from $\mathbb{J}$ and $\mathbb{K}$ is needed to make a complete bases for cubic system, in which the unit vectors of Cartesian coordinates are the three crystallographic direction. Following Walpole [1984], a general symmetric fourth-order tensor for a cubic system can be given by a linear combination of the three linear independent

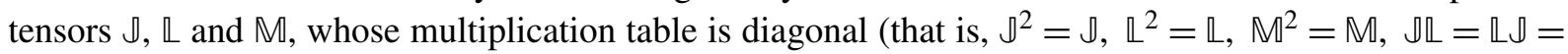
$\mathbb{I}=\mathbb{M} \mathbb{J}=\mathbb{L M}=\mathbb{M} \mathbb{L}=\mathbf{0}$ ). Using Kelvin notation, any cubic second-order tensor may be written in the form of

$$
\hat{\mathbf{A}}=a \hat{\mathbf{J}}+b \hat{\mathbf{L}}+c \hat{\mathbf{M}}
$$

where $\hat{\mathbf{J}}$ has been given in the previous section (equation (3-3)), while

$$
\begin{gathered}
\hat{\mathbf{L}}=\operatorname{diag}(0,0,0,1,1,1), \\
\hat{\mathbf{M}}=\hat{\mathbf{K}}-\hat{\mathbf{L}},
\end{gathered}
$$

using $\hat{\mathbf{K}}$ from (3-4). By writing $\hat{\mathbf{L}}$ in terms of $\hat{\mathbf{K}}$ and $\hat{\mathbf{M}}$, a general cubic elasticity tensor may be written in the form of linear combination of $\hat{\mathbf{J}}$, $\hat{\mathbf{K}}$ and $\hat{\mathbf{M}}$ as

$$
\hat{\mathbf{C}}_{\mathrm{cub}}=3 \kappa \hat{\mathbf{J}}+2 \mu \hat{\mathbf{K}}+2 \eta \hat{\mathbf{M}} \text {. }
$$

The closest cubic material using the conventional Euclidean distance was studied by Moakher and Norris [2006]. However, a different definition for the norm of a tensor was adopted in [Moakher 2006] where $\|\mathbf{A}\| \equiv\left[\operatorname{tr}\left(\mathbf{A}^{T} \mathbf{A}\right)\right]^{1 / 2}$. On the other hand, we continue using the previously defined distance functions in this section to determine the closest cubic system to the arbitrary anisotropic elasticity tensor. The derivation is quite similar to that of the previous section. The $f$-distance function from an arbitrary tensor to a cubic system is given as

$$
d_{f}=\left\|\boldsymbol{f}\left(\frac{\hat{\mathbf{C}}}{E_{0}}\right)-\boldsymbol{f}\left(\frac{\hat{\mathbf{C}}_{\mathrm{cub}}}{E_{0}}\right)\right\| .
$$

The results of applying the function $f$ on a cubic elastic tensor is also a linear combination of the bases [Moakher 2006]

$$
\boldsymbol{f}\left(\hat{\mathbf{C}}_{\text {cub }}\right)=f(3 \kappa) \hat{\mathbf{J}}+f(2 \mu) \hat{\mathbf{L}}+f(2 \eta) \hat{\mathbf{M}}
$$

In the same way, if we use the scalar gauge $E_{0}$, we may write

$$
\boldsymbol{f}\left(\frac{\hat{\mathbf{C}}_{\mathrm{cub}}}{E_{0}}\right)=f\left(\frac{3 \kappa}{E_{0}}\right) \hat{\mathbf{J}}+f\left(\frac{2 \mu}{E_{0}}\right) \hat{\mathbf{L}}+f\left(\frac{2 \eta}{E_{0}}\right) \hat{\mathbf{M}}
$$

Substituting (3-20) into (3-18) and looking for the stationarity points of $d_{f}$, or equally $d_{f}^{2}$, results in

$$
\frac{\partial\left(d_{f}^{2}\right)}{\partial \kappa}=0, \quad \frac{\partial\left(d_{f}^{2}\right)}{\partial \mu}=0, \quad \frac{\partial\left(d_{f}^{2}\right)}{\partial \eta}=0
$$

from which the following equations are derived:

$$
f\left(\frac{3 \kappa}{E_{0}}\right)=f\left(\frac{\hat{\mathbf{C}}}{E_{0}}\right): \hat{\mathbf{J}}, \quad f\left(\frac{2 \mu}{E_{0}}\right)=\frac{1}{3} f\left(\frac{\hat{\mathbf{C}}}{E_{0}}\right): \hat{\mathbf{L}}, \quad f\left(\frac{2 \eta}{E_{0}}\right)=\frac{1}{2} f\left(\frac{\hat{\mathbf{C}}}{E_{0}}\right): \hat{\mathbf{M}}
$$




\begin{tabular}{c|cccccc} 
& $\mathbb{E}_{1}$ & $\mathbb{E}_{2}$ & $\mathbb{E}_{3}$ & $\mathbb{E}_{4}$ & $\mathbb{F}$ & $\mathbb{G}$ \\
\hline $\mathbb{E}_{1}$ & $\mathbb{E}_{1}$ & $\mathbb{O}$ & $\mathbb{E}_{3}$ & $\mathbb{O}$ & $\mathbb{O}$ & $\mathbb{O}$ \\
$\mathbb{E}_{2}$ & $\mathbb{O}$ & $\mathbb{E}_{2}$ & $\mathbb{O}$ & $\mathbb{E}_{4}$ & $\mathbb{O}$ & $\mathbb{O}$ \\
$\mathbb{E}_{3}$ & $\mathbb{O}$ & $\mathbb{E}_{3}$ & $\mathbb{O}$ & $\mathbb{E}_{1}$ & $\mathbb{O}$ & $\mathbb{O}$ \\
$\mathbb{E}_{4}$ & $\mathbb{E}_{4}$ & $\mathbb{O}$ & $\mathbb{E}_{2}$ & $\mathbb{O}$ & $\mathbb{O}$ & $\mathbb{O}$ \\
$\mathbb{F}$ & $\mathbb{O}$ & $\mathbb{O}$ & $\mathbb{O}$ & $\mathbb{O}$ & $\mathbb{F}$ & $\mathbb{O}$ \\
$\mathbb{G}$ & $\mathbb{O}$ & $\mathbb{O}$ & $\mathbb{O}$ & $\mathbb{O}$ & $\mathbb{O}$ & $\mathbb{G}$
\end{tabular}

Table 1. Multiplication table for transversely isotropic tensors.

As mentioned earlier, the inverse of $f$-function exists, so that the scalar coefficients $\kappa, \mu$ and $\eta$ are derived from

$$
\kappa=\frac{E_{0}}{3} f^{-1}\left(f\left(\frac{\hat{\mathbf{C}}}{E_{0}}\right): \hat{\mathbf{J}}\right), \quad \mu=\frac{E_{0}}{2} f^{-1}\left(\frac{1}{3} f\left(\frac{\hat{\mathbf{C}}}{E_{0}}\right): \hat{\mathbf{L}}\right), \quad \eta=\frac{E_{0}}{2} f^{-1}\left(\frac{1}{2} f\left(\frac{\hat{\mathbf{C}}}{E_{0}}\right): \hat{\mathbf{M}}\right) .
$$

Again, here it is easy to show that the values of $\kappa, \mu$ and $\eta$ are independent from the gauge parameter $E_{0}$ for $\log$-distance function, while their values are dependent on $E_{0}$ for power-distance functions.

3C. Closest transversely isotropic material for arbitrary anisotropic material. Five linearly independent fourth-order tensors are needed to make a complete bases for a general fourth-order transversely isotropic tensor. Following Walpole [1984], we use the fourth-order tensors $\mathbb{E}_{1}, \mathbb{E}_{2}, \mathbb{E}_{3}+\mathbb{E}_{4}, \mathbb{F}$ and $\mathbb{G}$, where the summation of $\mathbb{E}_{3}$ and $\mathbb{E}_{4}$ comes due to symmetry of the elastic tensor, and the multiplication table was given in [Walpole 1984] (see also [Moakher and Norris 2006]). Thus, any arbitrary transversely isotropic elastic tensor using Kelvin notation is written in the form of the following linear combination of the bases

$$
\hat{\mathbf{C}}_{\mathrm{tr}}=a \hat{\mathbf{E}}_{\mathbf{1}}+b \hat{\mathbf{E}}_{\mathbf{2}}+c\left(\hat{\mathbf{E}}_{\mathbf{3}}+\hat{\mathbf{E}}_{\mathbf{4}}\right)+f \hat{\mathbf{F}}+g \hat{\mathbf{G}} .
$$

The multiplication Table 1 for $\mathbb{E}_{1}, \mathbb{E}_{2}, \mathbb{E}_{3}+\mathbb{E}_{4}, \mathbb{F}$ and $\mathbb{G}$ is not diagonal. Here $\mathbb{E}$ and $\mathbb{G}$ are orthogonal to each other and also orthogonal to $\mathbb{E}_{1}, \mathbb{E}_{2}$ and $\mathbb{E}_{3}+\mathbb{E}_{4}$. However $\mathbb{E}_{1}, \mathbb{E}_{2}$ and $\mathbb{E}_{3}+\mathbb{E}_{4}$ do not make an orthogonal set. This means that the scalar coefficients $a, b$ and $c$ are determined by solving a coupled system of equations, which is independent from $f$ and $g$. On the other hand, $f$ and $g$ are determined based on uncoupled equations.

To have explicit forms of the basic tensors $\hat{\mathbf{E}}_{\mathbf{1}}, \hat{\mathbf{E}}_{\mathbf{2}}, \hat{\mathbf{E}}_{\mathbf{3}}, \hat{\mathbf{E}}_{\mathbf{4}}, \hat{\mathbf{F}}$ and $\hat{\mathbf{G}}$, we use unit vectors $\hat{\mathbf{a}}, \hat{\mathbf{b}}$ and $\hat{\mathbf{c}}$ defined as

$$
\begin{aligned}
& \hat{\mathbf{a}}=\left(\begin{array}{llllll}
1 & 0 & 0 & 0 & 0 & 0
\end{array}\right)^{\mathbf{T}}=\left(a_{1}^{2}, a_{2}^{2}, a_{3}^{2}, \sqrt{2} a_{2} a_{3}, \sqrt{2} a_{3} a_{1}, \sqrt{2} a_{1} a_{2}\right)^{\mathbf{T}}, \\
& \hat{\mathbf{b}}=\left(\begin{array}{llllll}
0 & 1 & 0 & 0 & 0 & 0
\end{array}\right)^{\mathbf{T}}=\left(b_{1}^{2}, b_{2}^{2}, b_{3}^{2}, \sqrt{2} b_{2} b_{3}, \sqrt{2} b_{3} b_{1}, \sqrt{2} b_{1} b_{2}\right)^{\mathbf{T}}, \\
& \hat{\mathbf{c}}=\left(\begin{array}{llllll}
0 & 0 & 1 & 0 & 0 & 0
\end{array}\right)^{\mathbf{T}},
\end{aligned}
$$

to define the unit vectors

$$
\begin{aligned}
& \hat{\mathbf{p}}=\hat{\mathbf{c}}, \quad \hat{\mathbf{q}}=\frac{1}{\sqrt{2}}(\hat{\mathbf{a}}+\hat{\mathbf{b}}), \quad \hat{\mathbf{z}}=\frac{1}{\sqrt{2}}(\hat{\mathbf{a}}-\hat{\mathbf{b}}), \\
& \hat{\mathbf{w}}=\left(\sqrt{2} a_{1} b_{1}, \sqrt{2} a_{2} b_{2}, a \sqrt{2} a_{3} b_{3},\left(a_{2} b_{3}+a_{3} b_{2}\right),\left(a_{3} b_{1}+a_{1} b_{3}\right),\left(a_{1} b_{2}+a_{2} b_{1}\right)\right)^{\mathbf{T}},
\end{aligned}
$$


which are used to define the basic tensors as

$$
\hat{\mathbf{E}}_{1}=\hat{\mathbf{p}} \hat{\mathbf{p}}^{\mathbf{T}}, \quad \hat{\mathbf{E}}_{2}=\hat{\mathbf{q}} \hat{\mathbf{q}}^{\mathbf{T}}, \quad \hat{\mathbf{E}}_{3}=\hat{\mathbf{p}} \hat{\mathbf{q}}^{\mathbf{T}}, \quad \hat{\mathbf{E}}_{4}=\hat{\mathbf{q}} \hat{\mathbf{p}}^{\mathbf{T}}, \quad \hat{\mathbf{F}}=\hat{\mathbf{w}} \hat{\mathbf{w}}^{\mathbf{T}}+\hat{\mathbf{z}} \hat{\mathbf{z}}^{\mathbf{T}}, \quad \hat{\mathbf{G}}=\hat{\mathbf{L}}-\hat{\mathbf{w}} \hat{\mathbf{w}}^{\mathbf{T}},
$$

in which the $x_{3}$-axis is the axis of material symmetry of the transversely isotropic material defined in this way. $\hat{\mathbf{L}}$ in (3-27) can be found in (3-15).

Because of different coupled system of algebraic equations encountered in determining the closest transversely isotropic tensor to general arbitrary anisotropic material, we need to present the formulations for different distance functions separately.

3C1. The conventional Euclidean distance. Using the Euclidean distance, the distance function is defined as

$$
d_{C}=\left\|\hat{\mathbf{C}}-\hat{\mathbf{C}}_{\mathrm{tr}}\right\|
$$

for the tensor $\hat{\mathbf{C}}_{\text {tr }}$ as noted in (3-24). Consequently, the distance function in square becomes

$$
\begin{aligned}
d_{C}^{2} & =\left\|\hat{\mathbf{C}}-\hat{\mathbf{C}}_{\mathrm{tr}}\right\|^{2}=\left\|\hat{\mathbf{C}}-a \hat{\mathbf{E}}_{\mathbf{1}}-b \hat{\mathbf{E}}_{\mathbf{2}}-c\left(\hat{\mathbf{E}}_{\mathbf{3}}+\hat{\mathbf{E}}_{\mathbf{4}}\right)-f \hat{\mathbf{F}}-g \hat{\mathbf{G}}\right\|^{2} \\
& =\left(\hat{\mathbf{C}}-a \hat{\mathbf{E}}_{\mathbf{1}}-b \hat{\mathbf{E}}_{\mathbf{2}}-c\left(\hat{\mathbf{E}}_{\mathbf{3}}+\hat{\mathbf{E}}_{\mathbf{4}}\right)-f \hat{\mathbf{F}}-g \hat{\mathbf{G}}\right):\left(\hat{\mathbf{C}}-a \hat{\mathbf{E}}_{\mathbf{1}}-b \hat{\mathbf{E}}_{\mathbf{2}}-c\left(\hat{\mathbf{E}}_{\mathbf{3}}+\hat{\mathbf{E}}_{\mathbf{4}}\right)-f \hat{\mathbf{F}}-g \hat{\mathbf{G}}\right) \\
& =\hat{\mathbf{C}}: \hat{\mathbf{C}}-2 a \hat{\mathbf{C}}: \hat{\mathbf{E}}_{\mathbf{1}}-2 b \hat{\mathbf{C}}: \hat{\mathbf{E}}_{\mathbf{2}}-2 c \hat{\mathbf{C}}:\left(\hat{\mathbf{E}}_{\mathbf{3}}+\hat{\mathbf{E}}_{\mathbf{4}}\right)-2 f \hat{\mathbf{C}}: \hat{\mathbf{F}}-2 g \hat{\mathbf{C}}: \hat{\mathbf{G}} \\
& +a^{2}+b^{2}+2 c^{2}+2 f^{2}+2 g^{2} .
\end{aligned}
$$

The corresponding stationary points are obtained from

$$
\begin{aligned}
& \frac{\partial\left(d_{C}^{2}\right)}{\partial a}=2 a-2 \hat{\mathbf{C}}: \hat{\mathbf{E}}_{\mathbf{1}}=0, \quad \frac{\partial\left(d_{C}^{2}\right)}{\partial b}=2 b-2 \hat{\mathbf{C}}: \hat{\mathbf{E}}_{\mathbf{2}}=0, \quad \frac{\partial\left(d_{C}^{2}\right)}{\partial c}=4 c-2 \hat{\mathbf{C}}:\left(\hat{\mathbf{E}}_{\mathbf{3}}+\hat{\mathbf{E}}_{4}\right)=0, \\
& \frac{\partial\left(d_{C}^{2}\right)}{\partial f}=-2 \hat{\mathbf{C}}: \hat{\mathbf{F}}+4 f=0, \quad \frac{\partial\left(d_{C}^{2}\right)}{\partial g}=-2 \hat{\mathbf{C}}: \hat{\mathbf{G}}+4 g=0,
\end{aligned}
$$

resulting in

$$
a=\hat{\mathbf{C}}: \hat{\mathbf{E}}_{\mathbf{1}}, \quad b=\hat{\mathbf{C}}: \hat{\mathbf{E}}_{\mathbf{2}}, \quad c=\frac{1}{2} \hat{\mathbf{C}}:\left(\hat{\mathbf{E}}_{\mathbf{3}}+\hat{\mathbf{E}}_{\mathbf{4}}\right), \quad f=\frac{1}{2} \hat{\mathbf{C}}: \hat{\mathbf{F}}, \quad g=\frac{1}{2} \hat{\mathbf{C}}: \hat{\mathbf{G}} .
$$

3C2. The log-Euclidean distance. Here the log-distance function is used to find the closest transversely isotropic material for an arbitrary anisotropic elastic material. To do so, we may write the log-distance function as

$$
d_{L}=\left\|\ln \left(\frac{\hat{\mathbf{C}}}{E_{0}}\right)-\ln \left(\frac{\hat{\mathbf{C}_{t r}}}{E_{0}}\right)\right\| .
$$

From the definition of the logarithm of a positive definitive symmetric tensor $\hat{\mathbf{A}}$, one may write

$$
\ln (\beta \hat{\mathbf{A}})=\ln (\beta) \hat{\mathbf{I}}+\ln (\hat{\mathbf{A}}), \quad \forall \beta>0,
$$

which by virtue of the relation given in (3-32), results in

$$
d_{L}=\left\|\ln (\hat{\mathbf{C}})-\ln \left(\hat{\mathbf{C}}_{\mathrm{tr}}\right)\right\|
$$


With the use of (3-24) for the transversely isotropic material, we may write [Moakher and Norris 2006]

$$
\ln \hat{\mathbf{C}}_{\mathrm{tr}}=1_{1} \hat{\mathbf{E}}_{\mathbf{1}}+\mathrm{l}_{2} \hat{\mathbf{E}}_{\mathbf{2}}+\mathrm{l}_{3}\left(\hat{\mathbf{E}}_{\mathbf{3}}+\hat{\mathbf{E}}_{\mathbf{4}}\right)+\ln f \hat{\mathbf{F}}+\ln g \hat{\mathbf{G}},
$$

where the $1_{j}$ are introduced shortly.

Looking for the stationary points of $d_{L}^{2}$ with respect to $1_{1}, 1_{2}, 1_{3}, f$ and $g$, we reach to the following equations

$$
\begin{aligned}
& \frac{\partial\left(d_{L}^{2}\right)}{\partial \mathrm{l}_{1}}=2 \mathrm{l}_{1}-2 \ln (\hat{\mathbf{C}}): \hat{\mathbf{E}}_{\mathbf{1}}=0, \quad \frac{\partial\left(d_{L}^{2}\right)}{\partial \mathrm{l}_{2}}=2 \mathrm{l}_{2}-2 \ln (\hat{\mathbf{C}}): \hat{\mathbf{E}}_{\mathbf{2}}=0, \\
& \frac{\partial\left(d_{L}^{2}\right)}{\partial \mathrm{l}_{3}}=4 \mathrm{l}_{3}-2 \ln (\hat{\mathbf{C}}):\left(\hat{\mathbf{E}}_{\mathbf{3}}+\hat{\mathbf{E}}_{\mathbf{4}}\right)=0, \quad \frac{\partial\left(d_{L}^{2}\right)}{\partial f}=-\frac{2}{f} \ln (\hat{\mathbf{C}}): \hat{\mathbf{F}}+\frac{4}{f} \ln f=0, \\
& \frac{\partial\left(d_{L}^{2}\right)}{\partial g}=-\frac{2}{g} \ln (\hat{\mathbf{C}}): \hat{\mathbf{G}}+\frac{4}{g} \ln g=0,
\end{aligned}
$$

from which the following analytical solutions result in for the unknown parameters $1_{1}, 1_{2}, 1_{3}, \ln f$ and $\ln g$ :

$$
l_{1}=\ln (\hat{\mathbf{C}}): \hat{\mathbf{E}}_{\mathbf{1}}, \quad l_{2}=\ln (\hat{\mathbf{C}}): \hat{\mathbf{E}}_{\mathbf{2}}, \quad l_{3}=\frac{1}{2} \ln (\hat{\mathbf{C}}):\left(\hat{\mathbf{E}}_{\mathbf{3}}+\hat{\mathbf{E}}_{\mathbf{4}}\right), \quad \ln f=\frac{1}{2} \ln (\hat{\mathbf{C}}): \hat{\mathbf{F}}, \quad \ln g=\frac{1}{2} \ln (\hat{\mathbf{C}}): \hat{\mathbf{G}}
$$

From these five quantities we obtain $f$ and $g$. However, we need to determine the parameters $a, b, c$. According to [Walpole 1984], we have

$$
1_{1}=\ln \delta+\beta l, \quad 1_{2}=\ln \delta-\beta l, \quad 1_{3}=c l,
$$

in which

$$
l=\left\{\begin{array}{cl}
\frac{1}{\alpha} & \text { if } \gamma=0, \quad \alpha=\frac{1}{2}(a+b), \quad \gamma=\frac{1}{2} \sqrt{(a-b)^{2}+4 c^{2}} . \\
\frac{1}{2 \gamma} \ln \frac{\alpha+\gamma}{\alpha-\gamma} & \text { otherwise, }
\end{array}\right.
$$

Then, $a, b$ and $c$ can, at least numerically, be determined by solving the system of nonlinear equations just given. It is worth mentioning that $\gamma=0$ results in $a=b$ and $c=0$, which is a special transversely isotropic material. In this case, the bases is reduced to be constructed of four tensors $\mathbb{E}_{1}, \mathbb{E}_{2}, \mathbb{F}$ and $\mathbb{G}$, whose multiplication table is diagonal, and their sum come up to unit tensor.

Eventually, we may notice that there will be more than one solution and thus one has to choose the adequate solution.

3C3. The power-Euclidean distance. In this part, power-Euclidean distance functions are considered, from which the closest transversely isotropic tensor is given for an arbitrary anisotropic elastic material. We present the details for the cases $n=1$ and $n=0.5$.

(i) Power-Euclidean distance function with $n=1$

According to [Moakher and Norris 2006], the inverse of a transversely isotropic second-order tensor defined by (3-24) is given by

$$
\hat{\mathbf{C}}_{\mathrm{tr}}^{-1}=\frac{1}{a b-c^{2}}\left[b \hat{\mathbf{E}}_{\mathbf{1}}+a \hat{\mathbf{E}}_{\mathbf{2}}-c\left(\hat{\mathbf{E}}_{\mathbf{3}}+\hat{\mathbf{E}}_{\mathbf{4}}\right)\right]+\frac{1}{f} \hat{\mathbf{F}}+\frac{1}{g} \hat{\mathbf{G}} .
$$


According to the definition given in (2-10) for the power-Euclidean distance, we may write

$$
d_{P}=\left\|f\left(\frac{\hat{\mathbf{C}}}{E_{0}}\right)-\left(\frac{\hat{\mathbf{C}}_{\mathrm{tr}}}{E_{0}}\right)+\left(\frac{\hat{\mathbf{C}}_{\mathrm{tr}}}{E_{0}}\right)^{-1}\right\|,
$$

where

$$
f\left(\frac{\hat{\mathbf{C}}}{E_{0}}\right)=\left(\frac{\hat{\mathbf{C}}}{E_{0}}\right)-\left(\frac{\hat{\mathbf{C}}}{E_{0}}\right)^{-1}
$$

Substituting (3-24) and (3-40) into (3-41) results in

$$
\begin{array}{r}
d_{P}=\| \boldsymbol{f}\left(\frac{\hat{\mathbf{C}}}{E_{0}}\right)+\left(\frac{E_{0} b}{a b-c^{2}}-\frac{a}{E_{0}}\right) \hat{\mathbf{E}}_{\mathbf{1}}+\left(\frac{E_{0} a}{a b-c^{2}}-\frac{b}{E_{0}}\right) \hat{\mathbf{E}}_{\mathbf{2}}-\left(\frac{E_{0} c}{a b-c^{2}}+\frac{c}{E_{0}}\right)\left(\hat{\mathbf{E}}_{\mathbf{3}}+\hat{\mathbf{E}}_{\mathbf{4}}\right) \\
+\left(\frac{E_{0}}{f}-\frac{f}{E_{0}}\right) \hat{\mathbf{F}}+\left(\frac{E_{0}}{g}-\frac{g}{E_{0}}\right) \hat{\mathbf{G}} \|
\end{array}
$$

One might define

$$
i=\frac{E_{0} b}{a b-c^{2}}-\frac{a}{E_{0}}, \quad j=\frac{E_{0} a}{a b-c^{2}}-\frac{b}{E_{0}}, \quad k=\frac{E_{0} c}{a b-c^{2}}+\frac{c}{E_{0}}, \quad l=\frac{E_{0}}{f}-\frac{f}{E_{0}}, \quad m=\frac{E_{0}}{g}-\frac{g}{E_{0}},
$$

from which (3-43) becomes

$$
d_{P}=\left\|\boldsymbol{f}\left(\frac{\hat{\mathbf{C}}}{E_{0}}\right)+i \hat{\mathbf{E}}_{\mathbf{1}}+j \hat{\mathbf{E}}_{\mathbf{2}}-k\left(\hat{\mathbf{E}}_{\mathbf{3}}+\hat{\mathbf{E}}_{\mathbf{4}}\right)+l \hat{\mathbf{F}}+m \hat{\mathbf{G}}\right\|
$$

Thus, the square of the distance function is written as

$$
\begin{aligned}
d_{P}^{2}= & \boldsymbol{f}\left(\frac{\hat{\mathbf{C}}}{E_{0}}\right): \boldsymbol{f}\left(\frac{\hat{\mathbf{C}}}{E_{0}}\right)+2 i \boldsymbol{f}\left(\frac{\hat{\mathbf{C}}}{E_{0}}\right): \hat{\mathbf{E}}_{\mathbf{1}}+2 j \boldsymbol{f}\left(\frac{\hat{\mathbf{C}}}{E_{0}}\right): \hat{\mathbf{E}}_{\mathbf{2}}-2 k \boldsymbol{f}\left(\frac{\hat{\mathbf{C}}}{E_{0}}\right):\left(\hat{\mathbf{E}}_{\mathbf{3}}+\hat{\mathbf{E}}_{\mathbf{4}}\right) \\
& +2 l \boldsymbol{f}\left(\frac{\hat{\mathbf{C}}}{E_{0}}\right): \hat{\mathbf{F}}+2 m \boldsymbol{f}\left(\frac{\hat{\mathbf{C}}}{E_{0}}\right): \hat{\mathbf{G}}+i^{2}+j^{2}+2 k^{2}+2 l^{2}+2 m^{2}
\end{aligned}
$$

where the relations $\hat{\mathbf{E}}_{\mathbf{p}}: \hat{\mathbf{E}}_{\mathbf{q}}=\hat{\mathbf{E}}_{\mathbf{p}}: \hat{\mathbf{F}}=\hat{\mathbf{E}}_{\mathbf{p}}: \hat{\mathbf{G}}=\hat{\mathbf{F}}: \hat{\mathbf{G}}=0$ and $\hat{\mathbf{E}}_{\mathbf{p}}: \hat{\mathbf{E}}_{\mathbf{p}}=1$, with $\mathbf{p}$ and $\mathbf{q} \neq \mathbf{p}$ equal to 1 , 2,3 or 4 , and $\hat{\mathbf{F}}: \hat{\mathbf{F}}=\hat{\mathbf{G}}: \hat{\mathbf{G}}=2$ have been used. One may find the stationary points of $d_{P}^{2}$ as the solution of the following system of equations

$$
\begin{aligned}
& \frac{\partial\left(d_{P}^{2}\right)}{\partial i}=2 \boldsymbol{f}\left(\frac{\hat{\mathbf{C}}}{E_{0}}\right): \hat{\mathbf{E}}_{\mathbf{1}}+2 i=0, \quad \frac{\partial\left(d_{P}^{2}\right)}{\partial j}=2 \boldsymbol{f}\left(\frac{\hat{\mathbf{C}}}{E_{0}}\right): \hat{\mathbf{E}}_{\mathbf{2}}+2 j=0, \\
& \frac{\partial\left(d_{P}^{2}\right)}{\partial k}=-2 \boldsymbol{f}\left(\frac{\hat{\mathbf{C}}}{E_{0}}\right):\left(\hat{\mathbf{E}}_{\mathbf{3}}+\hat{\mathbf{E}}_{\mathbf{4}}\right)+4 k=0, \quad \frac{\partial\left(d_{P}^{2}\right)}{\partial l}=2 \boldsymbol{f}\left(\frac{\hat{\mathbf{C}}}{E_{0}}\right): \hat{\mathbf{F}}+4 l=0, \\
& \frac{\partial\left(d_{P}^{2}\right)}{\partial m}=2 \boldsymbol{f}\left(\frac{\hat{\mathbf{C}}}{E_{0}}\right): \hat{\mathbf{G}}+4 m=0,
\end{aligned}
$$


whose solutions for $i, j, k, l$ and $m$ are presented as

$$
\begin{array}{ll}
i=-\boldsymbol{f}\left(\frac{\hat{\mathbf{C}}}{E_{0}}\right): \hat{\mathbf{E}}_{\mathbf{1}}, \quad j=-\boldsymbol{f}\left(\frac{\hat{\mathbf{C}}}{E_{0}}\right): \hat{\mathbf{E}}_{\mathbf{2}}, \quad k=\frac{1}{2} \boldsymbol{f}\left(\frac{\hat{\mathbf{C}}}{E_{0}}\right):\left(\hat{\mathbf{E}}_{\mathbf{3}}+\hat{\mathbf{E}}_{\mathbf{4}}\right), \\
l=-\frac{1}{2} \boldsymbol{f}\left(\frac{\hat{\mathbf{C}}}{E_{0}}\right): \hat{\mathbf{F}}, \quad m=-\frac{1}{2} \boldsymbol{f}\left(\frac{\hat{\mathbf{C}}}{E_{0}}\right): \hat{\mathbf{G}} .
\end{array}
$$

After $i, j, k, l$ and $m$ are known, equations (3-44) can be used to solve for $a, b, c, f$, and $g$. As previously mentioned and seen from (3-44), the first three equations are coupled, while the remaining two are uncoupled.

(ii) Power-Euclidean distance function with $n=1 / 2$

Based on (2-14), we need both the square root and the inverse of the square root of the sought closest transversely isotropic tensor. Writing the general form of a transversely isotropic tensor as (3-24), its square root is given as [Moakher and Norris 2006]

$$
\hat{\mathbf{C}}_{\mathrm{tr}}^{1 / 2}=\frac{1}{\sqrt{2(\alpha+\delta)}}\left[(a+\delta) \hat{\mathbf{E}}_{\mathbf{1}}+(b+\delta) \hat{\mathbf{E}}_{\mathbf{2}}+c\left(\hat{\mathbf{E}}_{\mathbf{3}}+\hat{\mathbf{E}}_{\mathbf{4}}\right)\right]+\sqrt{f} \hat{\mathbf{F}}+\sqrt{g} \hat{\mathbf{G}}
$$

The parameters $\delta$ and $\alpha$ in (3-49) have been previously given as $\delta=\sqrt{a b-c^{2}}$ and $\alpha=\frac{1}{2}(a+b)$. The inverse of the square root of $\hat{\mathbf{C}}_{\text {tr }}$ is then given with the use of (3-40). According to the definition given for the power-Euclidean distance in (2-10), the distance between the tensors for $n=\frac{1}{2}$ is given by

$$
d_{P}=\left\|\boldsymbol{f}\left(\frac{\hat{\mathbf{C}}}{E_{0}}\right)-\left(\frac{\hat{\mathbf{C}}_{\mathrm{tr}}}{E_{0}}\right)^{1 / 2}+\left(\frac{\hat{\mathbf{C}}_{\mathrm{tr}}}{E_{0}}\right)^{-1 / 2}\right\|,
$$

in which $\left(\hat{\mathbf{C}}_{\mathrm{tr}} / E_{0}\right)^{1 / 2}=\left(1 / \sqrt{E_{0}}\right) \hat{\mathbf{C}}_{\mathrm{tr}}^{1 / 2}$. With the same procedure as given for $n=1$, one may find the stationary points of the square of this distance-function in terms of $i, j, k, l$ and $m$ as

$$
\begin{array}{ll}
i=-f\left(\frac{\hat{\mathbf{C}}}{E_{0}}\right): \hat{\mathbf{E}}_{\mathbf{1}}, \quad j=-\boldsymbol{f}\left(\frac{\hat{\mathbf{C}}}{E_{0}}\right): \hat{\mathbf{E}}_{\mathbf{2}}, \quad k=\frac{1}{2} f\left(\frac{\hat{\mathbf{C}}}{E_{0}}\right):\left(\hat{\mathbf{E}}_{\mathbf{3}}+\hat{\mathbf{E}}_{\mathbf{4}}\right), \\
l=-\frac{1}{2} f\left(\frac{\hat{\mathbf{C}}}{E_{0}}\right): \hat{\mathbf{F}}, \quad m=-\frac{1}{2} f\left(\frac{\hat{\mathbf{C}}}{E_{0}}\right): \hat{\mathbf{G}},
\end{array}
$$

where $i, j, k, l$ and $m$ are written in terms of $a, b, c, f$ and $g$ in the form of

$$
\begin{array}{ll}
i=\frac{(b+\delta) \sqrt{2 E_{0}(\alpha+\delta)}}{(a+\delta)(b+\delta)-c^{2}}-\frac{a+\delta}{\sqrt{2 E_{0}(\alpha+\delta)}}, & j=\frac{(a+\delta) \sqrt{2 E_{0}(\alpha+\delta)}}{(a+\delta)(b+\delta)-c^{2}}-\frac{b+\delta}{\sqrt{2 E_{0}(\alpha+\delta)}}, \\
k=\frac{c \sqrt{2 E_{0}(\alpha+\delta)}}{(a+\delta)(b+\delta)-c^{2}}+\frac{c}{\sqrt{2 E_{0}(\alpha+\delta)}}, & l=\sqrt{\frac{E_{0}}{f}}-\sqrt{\frac{f}{E_{0}}}, \quad m=\sqrt{\frac{E_{0}}{g}}-\sqrt{\frac{g}{E_{0}}} .
\end{array}
$$

By knowing $i, j, k, l$ and $m$ from (3-51) the coefficients $a, b, c, f$ and $g$ are, at least numerically, determined by (3-52). It is seen that the equations for $f$ and $g$ are uncoupled from the other equations. On the other hand, the coefficients $a, b$ and $c$ are determined from three nonlinear coupled equations. One needs to be careful of the extra and nonphysical solutions which may be appear from numerical procedure. 


\section{Numerical examples}

We illustrate two examples in this section to present the closest isotropic, cubic and transversely isotropic elastic tensors. First the results are based on an orthotropic material, and second based on an anisotropic material with a full elasticity tensor. The former case is perhaps of particular importance due to the amount of engineering applications, e.g., for composites. The results presented are for the different $f$-distance functions derived in the previous section; that is conventional, log- and power-Euclidean distances. The latter method is calculated using different power orders. For these power-Euclidean cases, the elastic modulus are functions of the scalar gauge $E_{0}$ and thus evaluated and plotted versus $E_{0}$.

4A. Orthotropic elasticity tensor. Consider an orthotropic material according to the elasticity tensor

$$
\widehat{\mathbf{C}}_{\text {orth }}=\left(\begin{array}{cccccc}
11.13 & 7.59 & 4.80 & 0 & 0 & 0 \\
7.59 & 14.08 & 6.03 & 0 & 0 & 0 \\
4.80 & 6.03 & 21.33 & 0 & 0 & 0 \\
0 & 0 & 0 & 9.82 & 0 & 0 \\
0 & 0 & 0 & 0 & 7.12 & 0 \\
0 & 0 & 0 & 0 & 0 & 4.82
\end{array}\right)(\mathrm{GPa})
$$

The material constants for higher symmetry approximations are presented in Tables $1-3$. Several behaviour, noted for the isotropic approximation by Morin et al. [2020], may here also be stated for cubic and transversely isotropic cases. In all cases, the results due to the conventional and log-Euclidean distance functions are independent of the scalar gauge $E_{0}$ as expected. The power-Euclidean cases with different power orders vary with the gauge value, where the level of variation increases with the power order. The constant log- and varying power-Euclidean curves almost cross at a certain gauge level for each elastic moduli. However, by detail inspection, these curves do not actually meet at a specific mathematical point for each modulus case. For all material parameters the conventional Euclidean solution represents upper bounds for the power-Euclidean case with $n=1$. This follow directly from the distance functions definitions in Section 2B for the special case when $E_{0}$ approaches 0.

For the isotropic and cubic cases shown in Figures 1-2, the variation among the material constants using different distance functions (and gauge values) are more pronounced when compared to the transversely isotropic case presented in Figure 3. This is somewhat expected as the difference among the various distance theories should be less pronounced when aiming at approximating an original material
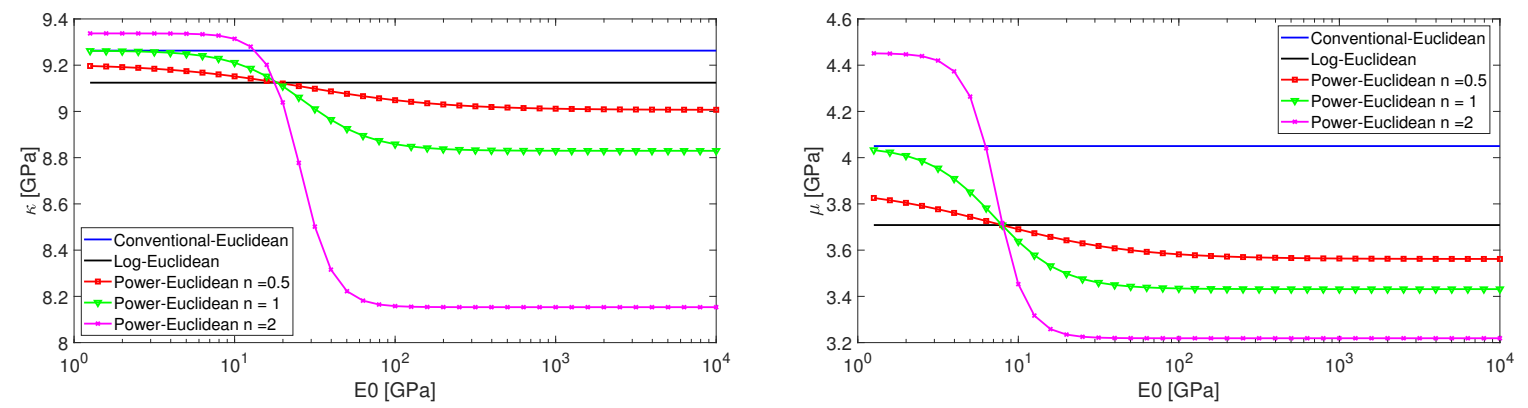

Figure 1. Closest isotropic tensors to (4-1): modulus $\kappa$ (left) and modulus $\mu$ (right). 

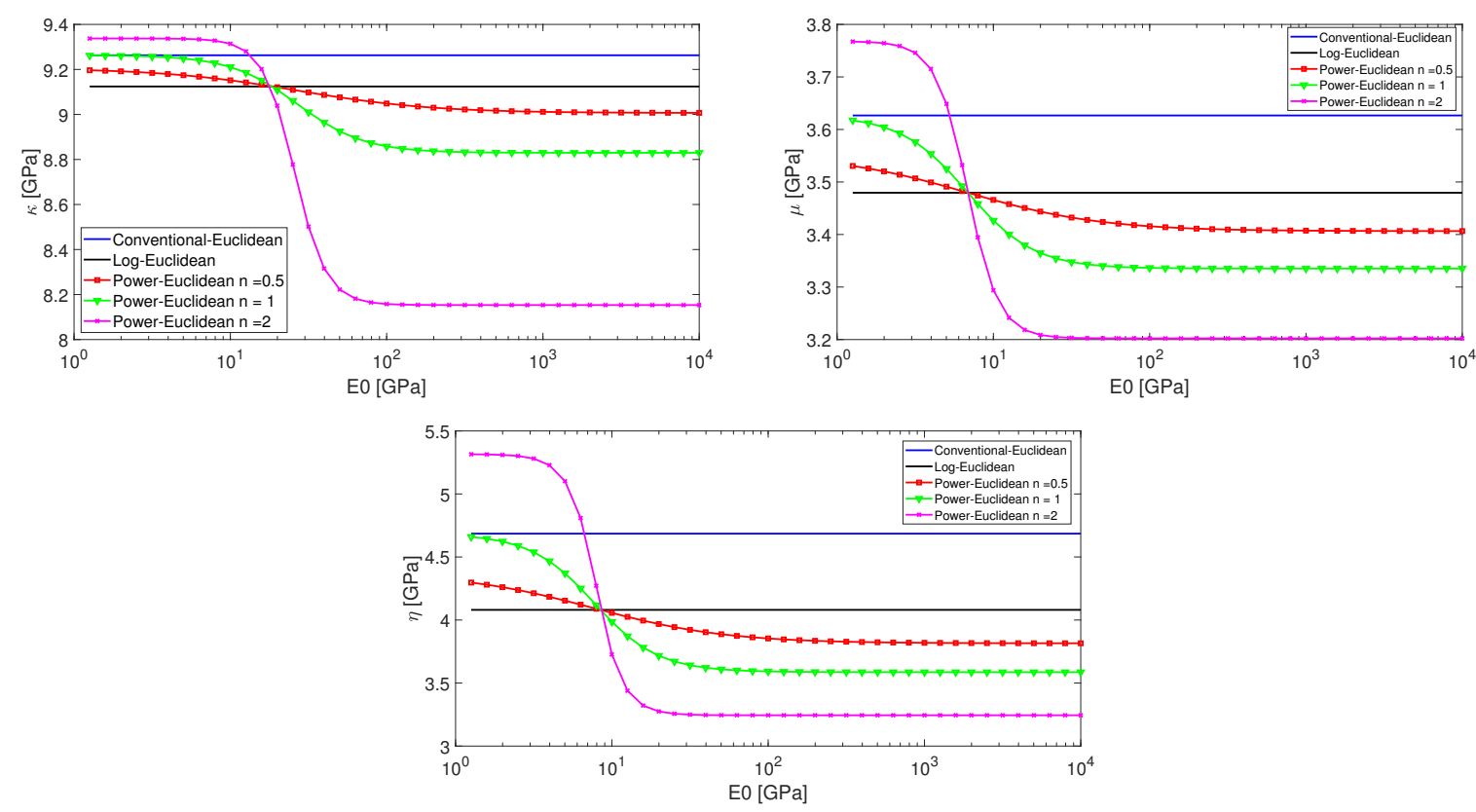

Figure 2. Closest cubic tensors to (4-1): modulus $\kappa$ (top left), modulus $\mu$ (top right), modulus $\eta$ (bottom).

(orthotropic) with an approximate material (transversely isotropic) with certain common symmetries. One may get an indication of the accuracies of each symmetry case by comparing the original tensor (4-1) to the elastic tensors given in the Appendix (Section 6A) for the log-Euclidean distance case.

A way to further study the accuracy for the higher symmetry approximations is to calculate and evaluate the error levels among the elasticity tensors using appropriate distance functions. Although one may chose among the distance functions presented in this paper, it is preferable to consider a somewhat alternative measure so as to avoid distinctly biased results. To this end, we here present results based on the Euclidean distance function for the eigenvalues $d_{C}\left(\widehat{\mathbf{D}}_{\text {orth }}, \widehat{\mathbf{D}}_{\text {orth,appr }}\right)$, where $\widehat{\mathbf{D}}$ is the diagonal eigenvalue matrix for an elastic tensor $\widehat{\mathbf{C}}$ see Section 2B. More specifically, normalized errors using $\left\|\widehat{\mathbf{D}}_{\text {orth }}-\widehat{\mathbf{D}}_{\text {orth,appr }}\right\| /\left\|\widehat{\mathbf{D}}_{\text {orth }}\right\|$ are presented for the closest isotropic, cubic and transversely isotropic elastic tensors based on the measures from log- and power-Euclidean distance functions, see Table 2. Here, the power cases with notation $(\mathrm{L})$ is for the material values using the scalar gauge lower limit $\left(E_{0} \rightarrow 0\right)$,

\begin{tabular}{c|lcccc} 
& $\log$ & $n=\frac{1}{2}(\mathrm{~L})$ & $n=\frac{1}{2}(\mathrm{H})$ & $n=1(\mathrm{~L})$ & $n=1(\mathrm{H})$ \\
\hline iso & 0.211 & 0.209 & 0.217 & 0.207 & 0.226 \\
cub & 0.178 & 0.166 & 0.195 & 0.149 & 0.212 \\
tr & 0.0564 & 0.0559 & 0.0571 & 0.0557 & 0.0594
\end{tabular}

Table 2. Normalized eigenvalue errors for higher symmetry approximations to (4-1) using material parameters based on different distance functions. 

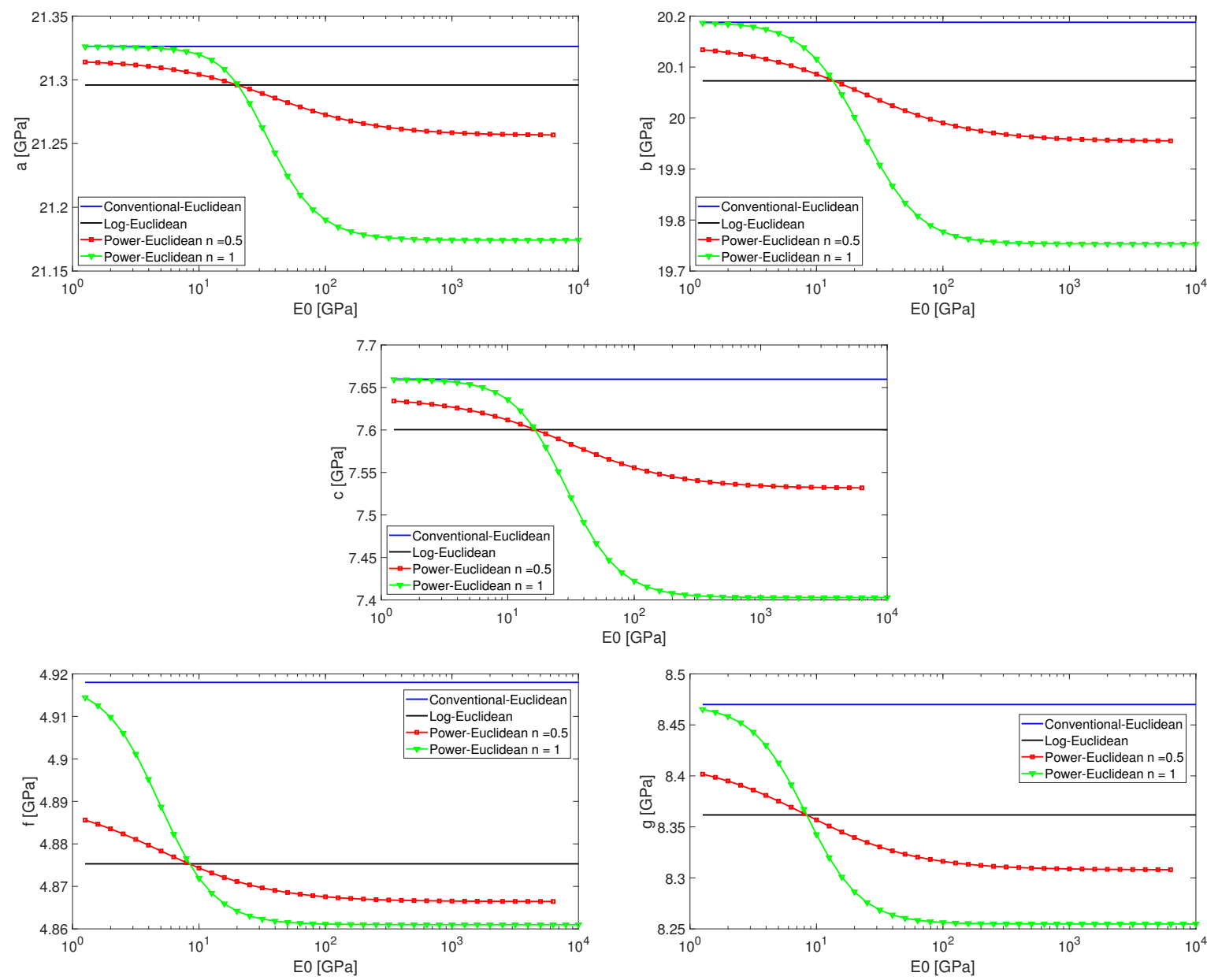

Figure 3. Closest transversely isotropic tensors to (4-1). Top row: moduli $a$ and $b$. Middle: modulus $c$. Bottom row: moduli $f$ and $g$.

while the notation $(\mathrm{H})$ is for the material values using the scalar gauge higher limit $\left(E_{0} \rightarrow \infty\right)$; see Figures 1-3.

These results show for each distance function measure that the isotropic approximation renders the largest errors as expected. Moreover, the transversely isotropic approximations results in considerably smaller errors compared to the cubic and isotropic approximations, in line with the expectations discussed above. Similar qualitative error estimates among isotropic, cubic and transversely isotropic approximations are obtained using other tested error norms besides Euclidean distance norm for the eigenvalues presented here. However, it should be emphasized that the best set of material parameters among the distance functions for a specific higher symmetry elastic tensor may not be stated here. The lowest errors for the power-Euclidean case $n=1$ (L) in Table 2 are related to the Euclidean eigenvalue norm adopted here.

4B. Full elasticity tensor. Consider next an anisotropic elasticity tensor studied by Morin et al. [2020]. 

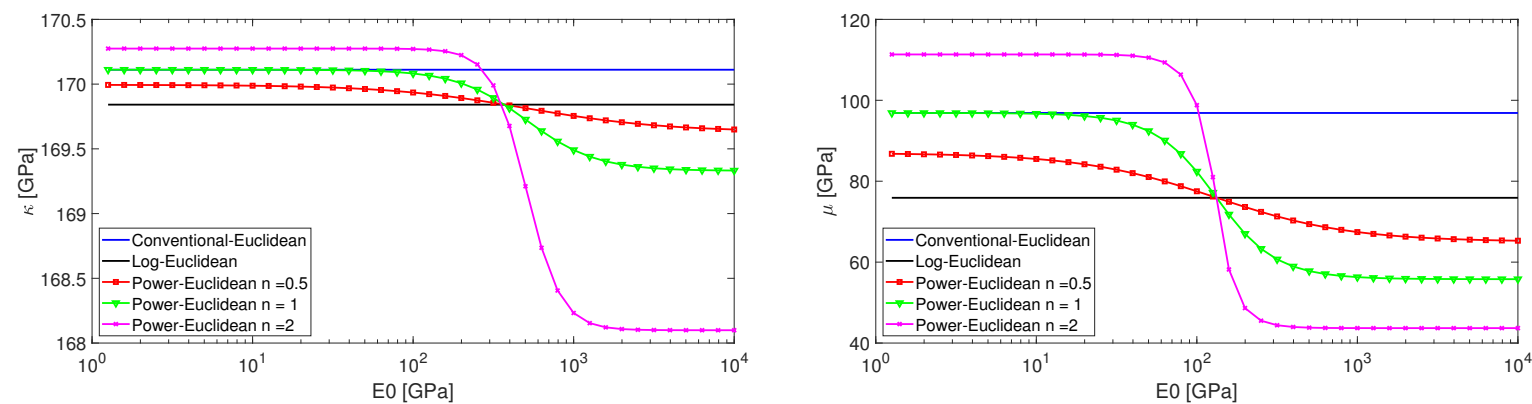

Figure 4. Closest isotropic tensors to (4-2): modulus $\kappa$ (left) and modulus $\mu$ (right).
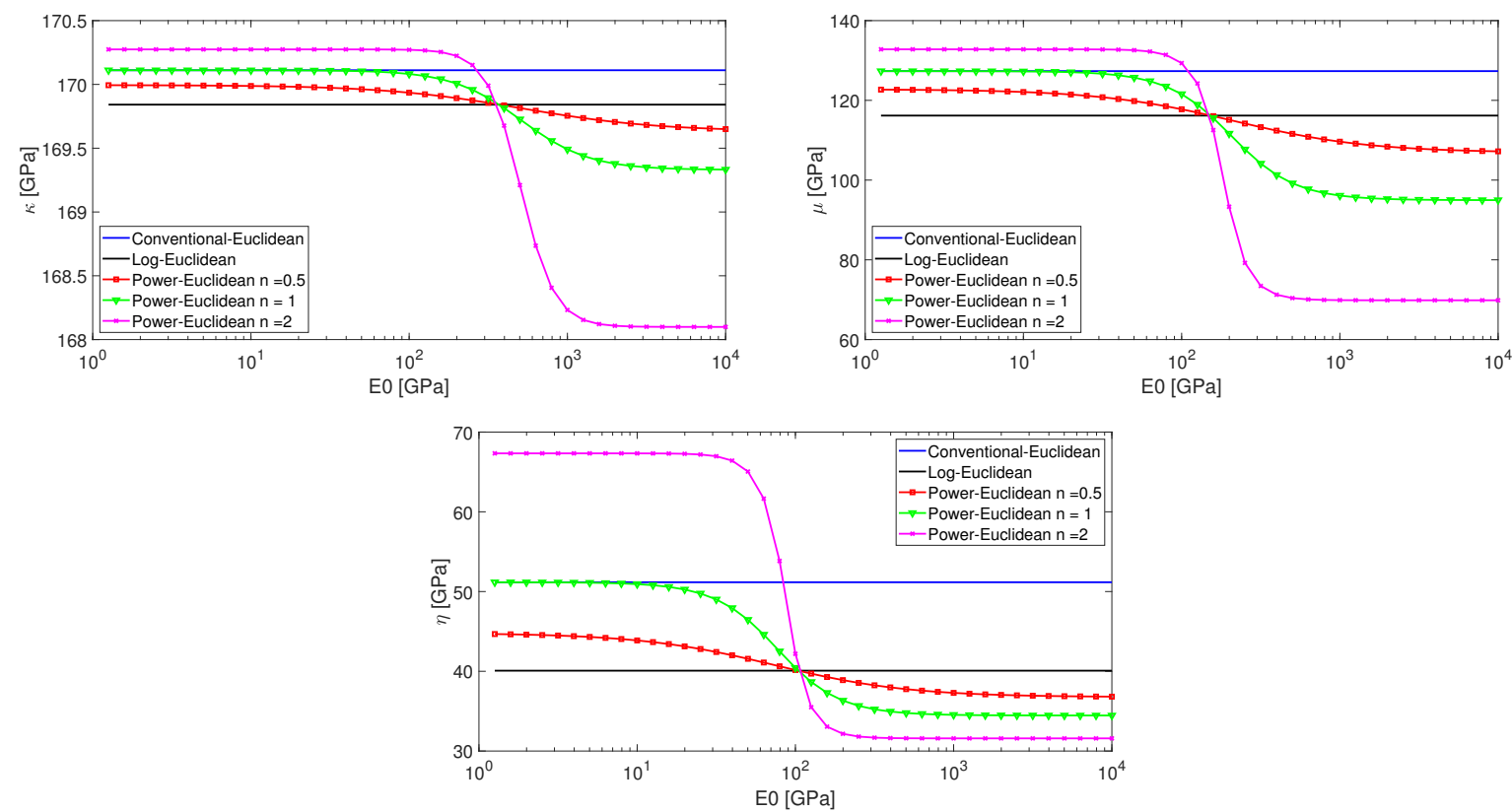

Figure 5. Closest cubic tensors to (4-2): modulus $\kappa$ (top left), modulus $\mu$ (top right), modulus $\eta$ (bottom).

This full anisotropic elasticity material parameters were determined from experiments by François et al. [1998]:

$$
\widehat{\mathbf{C}}_{\text {full }}=\left(\begin{array}{cccccc}
243 & 136 & 135 & 31.1 & 73.5 & -24 \\
136 & 239 & 137 & -39.6 & 15.6 & 22.6 \\
135 & 137 & 233 & 41 & -69.3 & 4.2 \\
31.1 & -39.6 & 41 & 266 & -20 & -4 \\
73.5 & 15.6 & -69.3 & -20 & 238 & -4 \\
-24 & 22.6 & 4.2 & -4 & -4 & 260
\end{array}\right)(\mathrm{GPa})
$$

The material constants for higher symmetry approximations are presented in Figures 4-6. Much of the behaviour reported for the higher symmetry approximations to orthotropic material in Section 4A also hold here. In addition the $c$ field in Figure 6, center, behaves differently compared to all the other 

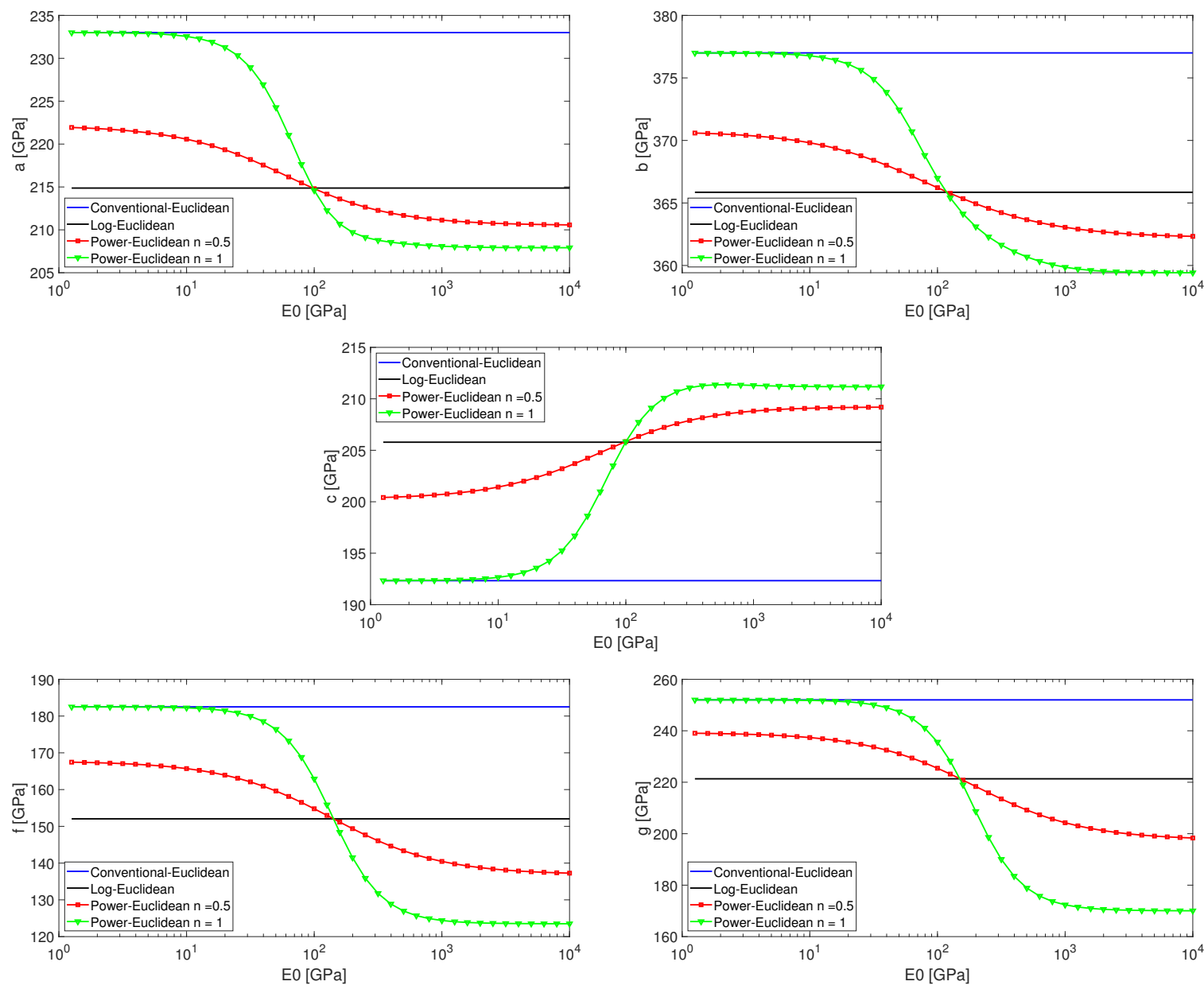

Figure 6. Closest transversely isotropic tensors to (4-2). Top row: moduli $a$ and $b$. Middle: modulus $c$. Bottom row: moduli $f$ and $g$.

fields as a function of the scalar gauge $E_{0}$ for the power-Euclidean cases. Here the parameter values increase with the gauge factor for reasons yet not explained.

The variation among the parameters for different distance functions (and gauge values) are here generally more pronounced compared to the orthotropic case presented in Section 4A. This could be seen as an indication that these more symmetric material configurations are probably less suitable to approximate the full anisotropic material presented here in (4-2). The corresponding approximate elastic tensors are expressed in the Appendix (Section 6B) for the log-Euclidean distance case.

The corresponding normalized eigenvalue errors $\left\|\widehat{\mathbf{D}}_{\text {full }}-\widehat{\mathbf{D}}_{\text {full,appr }}\right\| /\left\|\widehat{\mathbf{D}}_{\text {full }}\right\|$ are presented in Table 3 . As for the orthotropic case in Section 4A, the isotropic approximations render the largest errors as expected. However, the smallest errors are found for the cubic approximations for each distance function measure; a behaviour that may be supported by inspection of the tensors presented in Appendix 6B. Note that a 


\begin{tabular}{c|ccccc} 
& $\log$ & $n=\frac{1}{2}(\mathrm{~L})$ & $n=\frac{1}{2}(\mathrm{H})$ & $n=1(\mathrm{~L})$ & $n=1(\mathrm{H})$ \\
\hline iso & 0.366 & 0.348 & 0.395 & 0.342 & 0.427 \\
cub & 0.141 & 0.123 & 0.177 & 0.123 & 0.232 \\
tr & 0.230 & 0.211 & 0.265 & 0.209 & 0.314
\end{tabular}

Table 3. Normalized eigenvalue errors for higher symmetry approximations to (4-2) based on different distance functions.

cubic material is not a special case of transversely isotropic material. Moreover, the normalized error levels here are more pronounced compared to the orthotropic case (Table 2) as discussed above.

\section{Conclusion}

The closest fourth-order isotropic, cubic and transversely isotropic elasticity tensors to general anisotropic elasticity tensors have been analytically determined based on linear vector space and in the framework of Green elasticity. Different generalized Euclidean distance functions including conventional, log-, and power-Euclidean distance functions have been used. The power-Euclidean distance method has been illustrated for powers of 0.5, 1 and 2. A dimensionless procedure based on [Morin et al. 2020] has been used, and shown that the closest material for the power-Euclidean method is a function of the dimensionless parameter, while the conventional and log-Euclidean distance functions are independent from the dimensionless parameter.

The results from conventional Euclidean distance function are generally higher than from the logEuclidean distance function, and correspond to the extreme values for the power-Euclidean method with $n=1$. The material parameters using the log-Euclidean distance function are very close to the corresponding ones from the various power-Euclidean methods, provided the values where they almost intercept are chosen. In this way, one may proceed in line with Morin et al. [2020] and expect that the log-Euclidean distance function probably is generally the best choice among other distance functions used in this paper to represent the closest isotropic, cubic and transversely isotropic material to a general anisotropic material. However, this is yet to be proven.

The present work shows how to choose material constants based on various distance functions. In addition, it illustrates measures to evaluate among the higher symmetry tensors families for a specific base material. More specifically, the most appropriate symmetry class may be suggested such as adopting transversely isotropic symmetry for (4-1) and cubic symmetry for (4-2). However, the best choice among the distance functions (log, power, etc.) are not evaluated here. It should be noted that much remains regarding what simplification that should be stated for a specific engineering problem to secure a certain degree of accuracy. Possible future work is to evaluate both the influence from the choice of distance functions (log, power, etc.) and the chosen level of material symmetries (isotropic, cubic, transversely isotropic, etc.) for various standard engineering problems (both elastostatics and elastodynamics).

\section{Appendix: Closest elasticity tensors}

This appendix presents the approximate elasticity tensors discussed in Section 4 for higher symmetry approximations adopting the log-Euclidean distance function. 
6A. Orthotropic elasticity tensors. Approximations to the elasticity tensor (4-1) in Section 4A.

Isotropic material with $\kappa=9.12 \mathrm{GPa}, \mu=3.71 \mathrm{GPa}$

$$
\widehat{\mathbf{C}}_{\text {orth, iso }}=\left(\begin{array}{cccccc}
14.07 & 6.65 & 6.65 & 0 & 0 & 0 \\
6.65 & 14.07 & 6.65 & 0 & 0 & 0 \\
6.65 & 6.65 & 14.07 & 0 & 0 & 0 \\
0 & 0 & 0 & 7.42 & 0 & 0 \\
0 & 0 & 0 & 0 & 7.42 & 0 \\
0 & 0 & 0 & 0 & 0 & 7.42
\end{array}\right)(\mathrm{GPa})
$$

Cubic material with $\kappa=9.12 \mathrm{GPa}, \mu=3.48 \mathrm{GPa}, \eta=4.08 \mathrm{GPa}$

$$
\widehat{\mathbf{C}}_{\text {orth,cub }}=\left(\begin{array}{cccccc}
14.56 & 6.40 & 6.40 & 0 & 0 & 0 \\
6.40 & 14.56 & 6.40 & 0 & 0 & 0 \\
6.40 & 6.40 & 14.56 & 0 & 0 & 0 \\
0 & 0 & 0 & 6.96 & 0 & 0 \\
0 & 0 & 0 & 0 & 6.96 & 0 \\
0 & 0 & 0 & 0 & 0 & 6.96
\end{array}\right)(\mathrm{GPa})
$$

Transversely isotropic material with $a=21.30 \mathrm{GPa}, b=20.07 \mathrm{GPa}, c=7.60 \mathrm{GPa}, f=4.88 \mathrm{GPa}$, $g=8.36 \mathrm{GPa}$

$$
\widehat{\mathbf{C}}_{\text {orth }, \mathbf{t r}}=\left(\begin{array}{cccccc}
12.47 & 7.60 & 5.37 & 0 & 0 & 0 \\
7.60 & 12.47 & 5.37 & 0 & 0 & 0 \\
5.37 & 5.37 & 21.30 & 0 & 0 & 0 \\
0 & 0 & 0 & 8.36 & 0 & 0 \\
0 & 0 & 0 & 0 & 8.36 & 0 \\
0 & 0 & 0 & 0 & 0 & 4.88
\end{array}\right)(\mathrm{GPa})
$$

6B. Full elasticity tensors. Approximations to the elasticity tensor (4-2) in Section 4B.

Isotropic material with $\kappa=169.8 \mathrm{GPa}, \mu=75.9 \mathrm{GPa}$ :

$$
\widehat{\mathbf{C}}_{\text {full, iso }}=\left(\begin{array}{cccccc}
271.0 & 119.2 & 119.2 & 0 & 0 & 0 \\
119.2 & 271.0 & 119.2 & 0 & 0 & 0 \\
119.2 & 119.2 & 271.0 & 0 & 0 & 0 \\
0 & 0 & 0 & 151.8 & 0 & 0 \\
0 & 0 & 0 & 0 & 151.8 & 0 \\
0 & 0 & 0 & 0 & 0 & 151.8
\end{array}\right)(\mathrm{GPa})
$$

Cubic material with $\kappa=169.8 \mathrm{GPa}, \mu=116.2 \mathrm{GPa}, \eta=40.1 \mathrm{GPa}$ :

$$
\widehat{\mathbf{C}}_{\text {full,cub }}=\left(\begin{array}{cccccc}
223.3 & 143.1 & 143.1 & 0 & 0 & 0 \\
143.1 & 223.3 & 143.1 & 0 & 0 & 0 \\
143.1 & 143.1 & 223.3 & 0 & 0 & 0 \\
0 & 0 & 0 & 232.4 & 0 & 0 \\
0 & 0 & 0 & 0 & 232.4 & 0 \\
0 & 0 & 0 & 0 & 0 & 232.4
\end{array}\right)(\mathrm{GPa})
$$


Transversely isotropic material with $a=214.9 \mathrm{GPa}, b=365.9 \mathrm{GPa}, c=205.8 \mathrm{GPa}, f=152.0 \mathrm{GPa}$, $g=221.3 \mathrm{GPa}:$

$$
\widehat{\mathbf{C}}_{\text {full,tr }}=\left(\begin{array}{cccccc}
258.9 & 106.9 & 145.5 & 0 & 0 & 0 \\
106.9 & 258.9 & 145.5 & 0 & 0 & 0 \\
145.5 & 145.5 & 214.9 & 0 & 0 & 0 \\
0 & 0 & 0 & 221.3 & 0 & \\
0 & 0 & 0 & 0 & 221.3 & 0 \\
0 & 0 & 0 & 0 & 0 & 152.0
\end{array}\right)(\mathrm{GPa})
$$

\section{Acknowledgment}

The support from the University of Tehran to M. Eskandari-Ghadi during his sabbatical leave at the Chalmers University of Technology is greatly acknowledged. In addition, Eskandari-Ghadi acknowledges Prof. Peter D. Folkow and Prof. Reza Haghani for being his host professors during his sabbatical.

\section{References}

[Arsigny et al. 2005] V. Arsigny, P. Fillard, X. Pennec, and N. Ayache, "Fast and simple calculus on tensors in the log-Euclidean framework", pp. 115-122 in International Conference on Medical Image Computing and Computer-Assisted Intervention, Springer, 2005.

[Deza and Deza 2009] M. M. Deza and E. Deza, Encyclopedia of distances, Springer, 2009.

[François et al. 1998] M. François, G. Geymonat, and Y. Berthaud, "Determination of the symmetries of an experimentally determined stiffness tensor: application to acoustic measurements”, Int. J. Solids Struct. 35:31-32 (1998), 4091-4106.

[Gazis et al. 1963] D. C. Gazis, I. Tadjbakhsh, and R. A. Toupin, "The elastic tensor of given symmetry nearest to an anisotropic elastic tensor", Acta Crystall. 16 (1963), 917-922.

[Moakher 2006] M. Moakher, “On the Averaging of Symmetric Positive-Definite Tensors”, J. Elasticity 82:3 (2006), $273-296$.

[Moakher and Norris 2006] M. Moakher and A. N. Norris, "The Closest Elastic Tensor of Arbitrary Symmetry to an Elasticity Tensor of Lower Symmetry”, J. Elasticity 85:3 (2006), 215-263.

[Morin et al. 2020] L. Morin, P. Gilormini, and K. Derrien, "Generalized Euclidean distances for elasticity tensors", J. Elasticity 138:2 (2020), 221-232.

[Norris 2006a] A. Norris, "The isotropic material closest to a given anisotropic material", J. Mech. Mater. Struct. 1:2 (2006), $223-238$.

[Norris 2006b] A. N. Norris, "Elastic moduli approximation of higher symmetry for the acoustical properties of an anisotropic material”, J. Acoust. Soc. Am. 119:4 (2006), 2114-2121.

[Walpole 1984] L. J. Walpole, "Fourth-rank tensors of the thirty-two crystal classes: multiplication tables", Proc. R. Soc. Lond. A 391:1800 (1984), 149-179.

Received 14 Aug 2020. Revised 7 May 2021. Accepted 1 Jun 2021.

XinyUAn SHAO: shaox@student. chalmers.se

Applied Mechanics Masters Program, Chalmers University of Technology, 41296 Gothenburg, Sweden

PETER D. FOLKOW: peter.folkow@chalmers.se

Department of Mechanics and Maritime Sciences, Chalmers University of Technology, 41296 Gothenburg, Sweden

MORTEZA ESKANDARI-GHADI: m.eskandari@yahoo.com

School of Civil Engineering, University of Tehran, Tehran 1417466191, Iran 


\title{
JOURNAL OF MECHANICS OF MATERIALS AND STRUCTURES
}

\author{
msp.org/jomms
}

Founded by Charles R. Steele and Marie-Louise Steele

EDITORIAL BOARD

ADAIR R. AgUiAR

KATIA BERTOLDI

University of São Paulo at São Carlos, Brazil

DAVIDE BIGONI

MAENGHYO CHO

HUILING DUAN

YIBIN FU

IWONA JASIUK

DENNIS KOCHMANN

Mitsutoshi KURODA

CHEE W. LIM

ZISHUN LIU

Thomas J. Pence

GIANNI ROYER-CARFAGNI

DAVID STEIGMANN

Paul STEINMANN

Kenjiro Terada

Harvard University, USA

University of Trento, Italy

Seoul National University, Korea

Beijing University

Keele University, UK

University of Illinois at Urbana-Champaign, USA

ETH Zurich

Yamagata University, Japan

City University of Hong Kong

Xi' an Jiaotong University, China

Michigan State University, USA

Università degli studi di Parma, Italy

University of California at Berkeley, USA

Friedrich-Alexander-Universität Erlangen-Nürnberg, Germany

Tohoku University, Japan

ADVISORY BOARD

J. P. CARTER

D. H. HODGES

University of Sydney, Australia

Georgia Institute of Technology, USA

J. HutChInson Harvard University, USA

D. PAmplona Universidade Católica do Rio de Janeiro, Brazil

M. B. RUBIN Technion, Haifa, Israel

PRODUCTION production@msp.org

SILVIO LEVY Scientific Editor

Cover photo: Mando Gomez, www.mandolux.com

See msp.org/jomms for submission guidelines.

JoMMS (ISSN 1559-3959) at Mathematical Sciences Publishers, 798 Evans Hall \#6840, c/o University of California, Berkeley, CA 94720-3840, is published in 10 issues a year. The subscription price for 2021 is US \$660/year for the electronic version, and $\$ 830 /$ year (+\$60, if shipping outside the US) for print and electronic. Subscriptions, requests for back issues, and changes of address should be sent to MSP.

JoMMS peer-review and production is managed by EditFLOW ${ }^{\circledR}$ from Mathematical Sciences Publishers.

PUBLISHED BY

- mathematical sciences publishers

nonprofit scientific publishing

http://msp.org/

(C) 2021 Mathematical Sciences Publishers 


\title{
Journal of Mechanics of Materials and Structures
}

\author{
Volume 16, No. $4 \quad$ July 2021
}

Lamb waves in nonlocal elastic plate with voids

Gurwinderpal KaUr, Dilbag Singh and S. K. Tomar

Analysis on the interfacial mechanics of microscratching test for DLC-G95Cr18

film-substrate system Lixia Ying, Chong Yang Nie, Jianjun YaO, LE GU, YANG GE and LINING LIU

Quantifying fatigue cracks in TC4 titanium alloy using a nonlinear modulation ultrasonic testing method

Zhenhua Chen, Chenggeng Li, Guochen Xu and ChaO Lu

Analytical investigation on prediction of fatigue crack growth lives of cracked

nonhomogeneous materials

OĞUZHAN DEMIR

Degenerate orthotropic materials in the Stroh formalism for steady state problems

XU WANG and Peter Schiavone

The closest isotropic, cubic and transversely isotropic stiffness and compliance tensor to an arbitrary anisotropic material

Xinyuan Shao, Peter D. Folkow and Morteza Eskandari-Ghadi

The equivalent Havriliak-Negami model for characterizing the dynamic properties of viscoelastic dampers QIAng-QIang LI, ZhaO-Dong XU, YaO-ROng Dong, Zhen-HuA He, Yeshou Xu and Chen ZHU

The evolution of intragranular microcracks caused by interface migration induced by electromigration

Huang Shiqian and Huang Peizhen

Refining the thermoplasticity theory for modeling of cyclic nonisothermic loading

processes

VALENTIN S. BONDAR and DMITRY R. ABASHEV

Stress-smoothing holes in a regularly perforated elastic plate with a given effective bulk modulus

SHMUEL VIGDERGAUZ and ISAAC ELISHAKOFF

Free vibration of functionally graded piezoelectric hexagonal quasicrystal plates

Jinming Zhang, Liangliang Zhang, Yang Li, YunZhi Huang, Han

ZHANG and YANG GAO

Explicit model for bending edge wave on an elastic orthotropic plate supported by the Winkler-Fuss foundation

SaAd N. Althobaiti, Anatolij Nikonov and Danila Prikazchikov

Differential quadrature and Adomian decomposition methods for solving thermal vibration of Euler nanobeam resting on Winkler-Pasternak foundation SOMNATH KaRMAKAR and SNEHASHISH CHAKRAVERTY

Two-dimensional frictionless contact analysis of an orthotropic layer under gravity ERDAL ÖNER 